\title{
Static correlation and electron localization in molecular dimers from the self-consistent RPA and $G W$ approximation
}

\author{
Maria Hellgren,,${ }^{1,2}$ Fabio Caruso, ${ }^{3}$ Daniel R. Rohr, ${ }^{4}$ Xinguo Ren, ${ }^{5}$ Angel Rubio, ${ }^{6,4,7}$ Matthias Scheffler, ${ }^{7}$ and Patrick Rinke ${ }^{7,8}$ \\ ${ }^{1}$ International School for Advanced Studies (SISSA), via Bonomea 265, 34136 Trieste, Italy \\ ${ }^{2}$ Physics and Materials Science Research Unit, University of Luxembourg, 162a avenue de la Faïencerie, L-1511 Luxembourg, Luxembourg \\ ${ }^{3}$ Department of Materials, University of Oxford, Parks Road, Oxford OX1 3PH, United Kingdom \\ ${ }^{4}$ Nano-Bio Spectroscopy Group and European Theoretical Spectroscopy Facility (ETSF), Universidad del País Vasco CFM \\ CSIC-UPV/EHU-MPC DIPC, 20018 San Sebastián, Spain \\ ${ }^{5}$ Key Laboratory of Quantum Information, University of Science and Technology of China, Hefei, 230026, China \\ ${ }^{6}$ Max Planck Institute for the Structure and Dynamics of Matter, Hamburg, Germany \\ ${ }^{7}$ Fritz-Haber-Institut der Max-Planck-Gesellschaft, Faradayweg 4-6, D-14195 Berlin, Germany \\ ${ }^{8}$ COMP/Department of Applied Physics, Aalto University, P.O. Box 11100, Aalto FI-00076, Finland \\ (Received 23 December 2014; revised manuscript received 16 March 2015; published 7 April 2015)
}

\begin{abstract}
We investigate static correlation and delocalization errors in the self-consistent $G W$ and random-phase approximation (RPA) by studying molecular dissociation of the $\mathrm{H}_{2}$ and $\mathrm{LiH}$ molecules. Although both approximations contain topologically identical diagrams, the nonlocality and frequency dependence of the $G W$ self-energy crucially influence the different energy contributions to the total energy as compared to the use of a static local potential in the RPA. The latter leads to significantly larger correlation energies, which allow for a better description of static correlation at intermediate bond distances. The substantial error found in $G W$ is further analyzed by comparing spin-restricted and spin-unrestricted calculations. At large but finite nuclear separation, their difference gives an estimate of the so-called fractional spin error normally determined only in the dissociation limit. Furthermore, a calculation of the dipole moment of the $\mathrm{LiH}$ molecule at dissociation reveals a large delocalization error in $G W$ making the fractional charge error comparable to the RPA. The analyses are supplemented by explicit formulas for the $G W$ Green's function and total energy of a simplified two-level model providing additional insights into the dissociation limit.
\end{abstract}

DOI: 10.1103/PhysRevB.91.165110

PACS number(s): 71.10.-w, 31.15.E-

\section{INTRODUCTION}

Many-body perturbation theory (MBPT) [1] and KohnSham (KS) density functional theory (DFT) [2-4] are established as two prominent frameworks in computational electronic structure theory. Both are, in principle, exact but in practical calculations a careful choice of approximations has to be made. The central quantity to approximate is the exchange-correlation (xc) energy. In MBPT, the xc energy is calculated from the interacting one-particle Green's function $G$, which is obtained from the nonlocal and frequencydependent self-energy $(\Sigma)$ via Dyson's equation. In contrast, KS-DFT requires a local (i.e., multiplicative) and static exchange-correlation potential $\left(v_{\mathrm{xc}}\right)$ and hence approximations to the $\mathrm{xc}$ energy must be expressed in terms of a noninteracting KS Green's function. Only in their simplest form when both $v_{\mathrm{xc}}$ and $\Sigma$ are zero, i.e., in the Hartree approximation, the MBPT and KS-DFT formalisms are equivalent.

When more advanced approximations to the electronelectron interaction are considered, the nonlocality and frequency dependence of the self-energy-as opposed to the local and static xc potential in KS theory-may lead to qualitative differences in the description of the ground state and xc energy in MBPT and DFT. Understanding the origin of these differences is essential for advancing the development of new density functional and self-energy approximations, and it constitutes the main purpose of this work.

The $G W$ approximation [5] to MBPT and the random phase approximation (RPA) to DFT [6] are state-of-the-art approximations for first-principles excited- and ground-state electronic structure calculations [7-12]. Previous works have established their analogies and differences [13,14]. The xc energy in $G W$ and RPA can be represented in terms of topologically identical Feynman diagrams. Moreover, $G W$ and RPA share a common total-energy expression $[13,15,16]$, i.e., an expression with the same functional dependence on the single-particle Green's function. However, as alluded to in the first paragraph, the RPA energy is optimized with respect to Green's functions originating from a local KS potential, whereas the $G W$ energy is optimized by allowing for free variations of the Green's function leading to a nonlocal and frequency-dependent self-energy. The differences between RPA and $G W$ can thus be ascribed to the framework in which they are evaluated.

The performance of perturbative RPA (i.e., the RPA evaluated at a non-self-consistent KS Green's function) has been studied in several works [17-22]. Conversely, the $G W$ approximation, which has mostly been used for quasiparticle calculations, has only recently been explored for the calculation of ground-state properties $[23,24]$. The development of the fully self-consistent RPA (scRPA) and $G W(\operatorname{sc} G W)$ provides a unique assessment and evaluation of ground-state properties unbiased by the starting-point dependence that characterizes the perturbative approaches [13,25-29]. Moreover, self-consistency is essential to investigate the impact of advanced xc approximations on the electron density, since perturbative approaches do not alter the underlying wave function [30]. 
The total-energy curve of covalently-bonded diatomic molecules provides an important and difficult test case for both MBPT and DFT approximations. The dissociation of molecules with open-shell atoms such as $\mathrm{H}_{2}$ and $\mathrm{LiH}$ are characterized by a large degree of static correlation, i.e., the electronic wave function is not representable in terms of a single Slater determinant. Therefore the accuracy achieved in the description of the dissociation region reflects the capability of a given xc approximation to capture (or mimic) the multiple Slater determinant character of the wave function. It is already well known that RPA yields a good description of the total energy in the dissociation region [31-34], whereas $G W$ overestimates it considerably $[14,26]$. In addition to the problem of static correlation, dissociation tests the ability of a functional to localize the electrons, important to accurately capture the abrupt change in the density upon atomization. How well $G W$ and RPA perform in this regard is still unknown and will be addressed here.

In this paper, we present a thorough analysis of $\operatorname{sc} G W$ and scRPA for ground-state properties of diatomic molecules at dissociation. We investigate the impact of locality and nonlocality on the different energy contributions to the total energy. We also illustrate that a nonlocal treatment of exchange and correlation in the $G W$ approximation opens the gap between the highest-occupied and lowest-unoccupied molecular orbitals (HOMO and LUMO). Furthermore, the frequency dependence of the $G W$ self-energy is shown to significantly modify the ground-state of the system, leading, e.g., to a density matrix with fractional occupation numbers. Although $G W$ can capture important many-body physics it reaches very slowly to the dissociation limit. The local RPA potential, on the other hand, approaches the dissociation limit faster and thus yields a better description of the ground state energy in the dissociation region.

A convenient way to test the performance of an approximate functional with respect to static correlation and the ability to localize the electrons is to determine the so-called fractional spin and fractional charge errors. The fractional spin and fractional charge errors of the RPA have been studied previously $[33,35]$. In order to estimate the same errors in $G W$, we have used a more indirect approach. To determine an approximate fractional spin error, we compare spin-unrestricted dissociation with spin-restricted dissociation and in order to determine a fractional charge error we study the dipole moment of $\mathrm{LiH}$ as a function of nuclear separation. In this way, the errors are not determined in the dissociation limit but at large finite $R$, far beyond the point of atomization. Both $G W$ and RPA are affected by rather large fractional charge errors and hence suffer from an insufficient ability to accurately localize the electrons during dissociation. While RPA is free from fractional spin error at large interatomic distances, $G W$ also exhibits a rather large fractional spin error.

Our first-principles calculations are complemented by an analytic derivation of explicit formulas for the Green's function and the correlation energy of a model two-level $\mathrm{H}_{2}$ molecule. This allows us to investigate the limit of infinite interatomic separation, which is not accessible by numerical studies. The results indicate that $G W$ and RPA are very similar in the dissociation limit but that $G W$ is subjected to a very slowly converging gap leading to a very different behavior in the dissociation region. The spurious local maximum characteristic of the RPA total energy curve could therefore also be present in $G W$, albeit largely extended.

The paper is organized as follows. In Sec. II, we introduce the $G W$ and RPA total energy functionals. In Sec. III, we discuss the problem of covalent bond dissociation and the impact of nonlocality and frequency dependence on the ground-state properties of the system. Section IV presents the derivation of an analytic expression for the one-shot $G W$ Green's function and for the $G W$ and RPA correlation energy in the dissociation limit for a simplified two-level model. We present an analysis of the fractional spin and fractional charge errors in MBPT in Sec. V. Finally, our summary and conclusions are presented in Sec. VI.

\section{THEORY}

The usual and most direct way of calculating the total energy from the single-particle Green's function $G$ is via the Galitskii-Migdal (GM) formula [36]:

$$
E_{\mathrm{tot}}[G]=T[G]+E_{\mathrm{ext}}[G]+E_{\mathrm{H}}[G]+E_{\mathrm{xc}}[G],
$$

in which $T$ denotes the kinetic energy, $E_{\text {ext }}$ the external potential energy, and $E_{\mathrm{H}}$ the Hartree energy. The exchangecorrelation (xc) energy

$$
E_{\mathrm{xc}}[G]=\int_{0}^{\infty} \frac{d \omega}{2 \pi} \operatorname{Tr}\{\Sigma(i \omega) G(i \omega)\},
$$

is determined from $\Sigma$, which is here defined as the self-energy minus the Hartree potential $v_{\mathrm{H}}(\mathbf{r})=\int d \mathbf{r}^{\prime} n\left(\mathbf{r}^{\prime}\right) v\left(\mathbf{r}-\mathbf{r}^{\prime}\right)$. The self-energy is also needed to compute $G$ via Dyson's equation

$$
G=G_{\mathrm{H}}+G_{\mathrm{H}} \Sigma[G] G .
$$

Notice that spatial and time coordinates have been suppressed in order to keep the notation light. The GM expression is nonvariational (i.e., $\delta E_{\text {tot }}[G] / \delta G \neq 0$ when $G$ is the solution of the Dyson equation). Therefore, when evaluating Eq. (1), it is necessary that $G$ is obtained from the iterative solution of Eq. (3) in order to get accurate results (see, e.g., Refs. [23,25]). In MBPT it is also possible to formulate energy functionals, which are variational with respect to $G$ [i.e., $\delta E_{\text {tot }}[G] / \delta G=0$ when $G$ satisfies Eq. (3)]. Several different kinds have been proposed [37-39] but the most simple is the one introduced by Klein [15]:

$$
E_{\mathrm{K}}[G]=-i \Phi[G]+i \operatorname{Tr}\left\{G G_{\mathrm{H}}^{-1}-1+\ln \left(-G^{-1}\right)\right\}+E_{\mathrm{H}} .
$$

The $\Phi$ functional is related to the self-energy by $\Sigma=\delta \Phi / \delta G$. It is easy to verify that in any approximate but $\Phi$-derivable self-energy $E_{\mathrm{K}}$ is stationary when $G$ obeys Dyson's equation. Furthermore, at the stationary point $E_{\mathrm{K}}$ equals the total energy obtained from the corresponding GM formula. However, the Klein functional is more advantageous than the GM expression when considering approximate Green's functions since the variational property of the Klein functional ensures that the total energy will be close to that evaluated with the self-consistent $G$. Thus the variational nature of the Klein functional can be employed to avoid the numerical complexity of the Dyson's equation, and at the same time obtain an energy 
close to the self-consistent one. The accuracy to which this can be achieved has been investigated in previous work [26] and will be highlighted also in the present work.

We will now specialize the discussion to two $\Phi$-derivable approximations: the Hartree-Fock/exact-exchange (HF/EXX) and the $G W / \mathrm{RPA}$ approximations. At the HF level, the $\Phi$ functional assumes the following form:

$$
\Phi=\frac{i}{2} \operatorname{Tr}\{G G v\}
$$

It can be easily verified that applying the functional derivative $\Sigma=\delta \Phi / \delta G$ to Eq. (5) yields the Fock self-energy $i G v$, which is nonlocal and frequency independent. The HF Green's function will thus take the form of a noninteracting Green's function. Evaluating Eq. (4) with an arbitrary noninteracting Green's function-here denoted $G_{s}$ —results in [39]

$$
E_{\mathrm{K}}=T_{s}+E_{\mathrm{ext}}+E_{\mathrm{H}}-i \Phi\left[G_{s}\right],
$$

where $T_{s}$ is the kinetic energy of noninteracting electrons. When $\Phi$ is given by Eq. (5) this is just the standard HF expression for the total energy. By comparing Eqs. (1) and (6), one sees that at the HF level and at a noninteracting $G_{S}$ the Klein expression and the GM formula coincide.

We can further restrict the Green's function $G_{s}$ to come from a local potential. In this way, Eq. (6) has the same form as the total energy in KS DFT and $-i \Phi\left[G_{s}\right]$ can be identified with the KS xc energy [39]. If the HF approximation is constrained to be evaluated with a $G_{s}$ from a local potential, the Klein functional reduces to the EXX functional. It is well known that when the EXX functional is optimized with respect to the local EXX potential—as opposed to the nonlocal HF self-energy-it produces an energy very similar to (and slightly higher than) HF. For example, the difference in total energy of atoms is around 10 parts per million [40]. The HF Klein functional is thus quite stable with respect to variations in the Green's function.

Let us now turn to the $G W$ approximation for which the self-energy is given by

$$
\Sigma=i G W=i G\left[v+v \chi^{\mathrm{H}} v\right],
$$

where

$$
\chi^{\mathrm{H}}=\chi_{0}+\chi_{0} v \chi^{\mathrm{H}}
$$

is the reducible polarizability in the Hartree approximation and $\chi_{0}=i G G$ is the zeroth order approximation to the irreducible polarizability. In the $G W$ approximation for $\Sigma$, the GM exchange-correlation energy in Eq. (2) reduces to

$$
E_{\mathrm{xc}}[G]=E_{\mathrm{x}}[G]+\int_{0}^{\infty} \frac{d \omega}{2 \pi} \operatorname{Tr}\left\{v\left[\chi^{\mathrm{H}}(i \omega)-\chi_{0}(i \omega)\right]\right\} .
$$

Equations (9) and (1) provide the explicit form of the GM energy in the $G W$ approximation. At self-consistency, the GM energy is equal to that obtained from the Klein functional. Therefore, due to the simplicity of the GM expression and its natural decomposition into different energy contributions it is more convenient to use the GM expression whenever the selfconsistent $G W$ Green's function is considered. All the selfconsistent results presented in the next sections correspond to the evaluation of the GM formula.
Let us now evaluate the Klein $G W$ functional with a noninteracting $G_{s}$. The $\Phi$-functional generating the $G W$ self-energy is equal to

$$
\Phi=\frac{1}{2} \operatorname{Tr}\{\ln (1+i v G G)\} .
$$

It can be easily verified by functional differentiation of Eq. (10) that $\Sigma=\delta \Phi / \delta G=i G W$. If evaluated with a noninteracting Green's function $G_{s}$, Eq. (10) takes the form of the RPA xc energy functional:

$$
\begin{aligned}
& \frac{1}{2} \operatorname{Tr}\left\{\ln \left(1+i v G_{s} G_{s}\right)\right\} \\
& \quad=E_{\mathrm{x}}\left[G_{s}\right]+\int_{0}^{\infty} \frac{d \omega}{2 \pi} \int_{0}^{1} d \lambda \operatorname{Tr}\left\{v\left[\chi_{\lambda}^{\mathrm{H}}(i \omega)-\chi_{s}(i \omega)\right]\right\}
\end{aligned}
$$

where $E_{\mathrm{x}}=\frac{i}{2} \operatorname{Tr} G_{s} G_{s} v$ is the Fock exchange energy, $\chi_{s}=$ $i G_{s} G_{s}$ is the irreducible polarizability (or noninteracting density response function) evaluated with the KS Green's function and $\chi_{\lambda}^{\mathrm{H}}$ is the reducible polarizability (or the Hartree density response function) of a system with a linearly scaled Coulomb interaction $\lambda v$, i.e.,

$$
\chi_{\lambda}^{\mathrm{H}}=\chi_{s}+\lambda \chi_{s} v \chi_{\lambda}^{\mathrm{H}} \text {. }
$$

The RPA energy is therefore just the $G W$ Klein functional evaluated with a noninteracting Green's function. If we compare Eqs. (1) and (9) to Eqs. (6) and (11), we see that apart from the input Green's function, the $G W$ and RPA energy expressions differ only by a coupling constant integral.

To facilitate the comparison and make a clear distinction between the $G W$ and the RPA energies, we will from now on denote the GM $G W$ correlation energy $U_{\mathrm{c}}^{G W}$ and the Klein RPA correlation energy $E_{\mathrm{c}}^{\mathrm{RPA}}$ :

$$
\begin{gathered}
U_{\mathrm{c}}^{G W}=\int_{0}^{\infty} \frac{d \omega}{2 \pi} \operatorname{Tr}\left\{v\left[\chi^{\mathrm{H}}(i \omega)-\chi_{0}(i \omega)\right]\right\}, \\
E_{\mathrm{c}}^{\mathrm{RPA}}=\int_{0}^{\infty} \frac{d \omega}{2 \pi} \int_{0}^{1} d \lambda \operatorname{Tr}\left\{v\left[\chi_{\lambda}^{\mathrm{H}}(i \omega)-\chi_{s}(i \omega)\right]\right\} .
\end{gathered}
$$

Differently from $U_{\mathrm{c}}, E_{\mathrm{c}}$ incorporates also the so-called kinetic correlation energy-defined as the difference between the kinetic energy of the interacting system and that of the fictitious noninteracting particle system-which is included through the adiabatic-connection integrations over the interaction strength $\lambda$. $U_{\mathrm{c}}$, on the other hand, includes only electronic correlation arising from the Coulomb interaction. It is therefore possible to define the kinetic correlation energy in the RPA through the following expression:

$$
T_{\mathrm{c}}^{\mathrm{RPA}}\left[G_{s}\right] \equiv E_{\mathrm{c}}^{\mathrm{RPA}}\left[G_{s}\right]-U_{\mathrm{c}}^{G W}\left[G_{s}\right] .
$$

When the Klein and the GM functionals are evaluated with a noninteracting Green's function, the total energies obtained from the two approaches will differ exactly by $T_{\mathrm{c}}$, since the remaining energy terms in the Klein and GM formula have the same functional dependence on $G_{s}$. Using Eq. (15), we can rewrite the RPA total energy as

$$
\begin{aligned}
E^{\mathrm{RPA}}\left[G_{s}\right]= & T_{s}\left[G_{s}\right]+T_{c}^{\mathrm{RPA}}\left[G_{s}\right]+E_{\mathrm{ext}}\left[G_{s}\right] \\
& +E_{\mathrm{H}}\left[G_{s}\right]+E_{\mathrm{x}}\left[G_{s}\right]+U_{\mathrm{c}}^{G W}\left[G_{s}\right] .
\end{aligned}
$$


Notice again that this rewriting of the Klein functional as a functional of $G_{s}$ is only possible for a noninteracting $G_{s}$. The GM expression instead is defined for any $G$ and we will call this the $G W$ energy

$$
\begin{aligned}
E^{G W}[G]= & T[G]+E_{\mathrm{ext}}[G]+E_{\mathrm{H}}[G] \\
& +E_{\mathrm{x}}[G]+U_{\mathrm{c}}^{G W}[G] .
\end{aligned}
$$

In the next section, we will use Eqs. (16) and (17) for comparing the different energy contribution in the $G W$ and the RPA. In the following, we evaluate the Klein and GM functionals employing Green's functions evaluated in the RPA and $G W$ approximations. To avoid possible dependencies on the starting point, both approaches are iterated to full self-consistency-denoted as self-consistent $G W(\operatorname{sc} G W)$ and self-consistent RPA (scRPA). In $\operatorname{sc} G W$ the Green's function is obtained from the iterative solution of Dyson's equation (3) with $\Sigma$ in the $G W$ approximation [see Eq. (7)]. The sc $G W$ method has been implemented in the FHI-aims code $[43,44]$. Details of the $\operatorname{sc} G W$ implementation can be found elsewhere [45]. If not otherwise stated, coupled-cluster singles doubles (CCSD) and full configuration interaction (full-CI) calculations have been performed with the ORCA code package [46] using Dunning's cc-PVDZ and cc-PVTZ Gaussian basis sets [47] and extrapolated to the complete basis set limit.

In scRPA, the energy is optimized with respect to a local potential determined by the optimized effective potential equation (also known as the linearized Sham-Schlüter (LSS) equation) $[48,49]$. In RPA, it is given by $[13,50]$

$$
\int \chi_{s} v_{\mathrm{xc}}^{\mathrm{RPA}}=-i \int \frac{d \omega}{2 \pi} \Sigma^{G W}\left[G_{s}\right](\omega) G_{s}(\omega) G_{s}(\omega),
$$

where spacial variables have been suppressed. Details on the implementation can be found in Ref. [35].

For comparison, we will also consider the GM and Klein functional evaluated using Green's functions from the HF approximation and the semilocal PBE approximation [51]. In these cases, the corresponding GM (Klein) total energy functional is equivalent to the inclusion of $G W$ (RPA) correlation energy $U_{\mathrm{c}}^{G W}\left(E_{\mathrm{c}}^{\mathrm{RPA}}\right)$ at the level of first-order perturbation theory. This allows us to establish a connection with previous works in which the RPA and $G W$ total energies have been considered at a perturbative level.

\section{TOTAL ENERGY}

In this section, we present an analysis of the different contributions to the $\mathrm{H}_{2}$ total energy curve. The results illustrate how a nonlocal and frequency-dependent treatment of exchange and correlation effects may affect the ground-state properties of diatomic molecules composed of open-shell atoms.

\section{A. Coulomb energy and the HOMO-LUMO gap}

In Fig. 1, we report the dissociation curves of $\mathrm{H}_{2}$ evaluated with sc $G W$, scRPA, and HF. Exact reference values from full-CI calculations are included for comparison [41]. Due to the variational properties of the Klein functional, one may expect that $\operatorname{sc} G W$ and scRPA provide a qualitatively similar description of the total energy along the entire dissociation curve. However, as observed in Ref. [14], the sc $G W$ and scRPA total energies differ considerably for bond lengths larger than $2 \AA$.

In the following, we illustrate that the Coulomb correlation energy depends significantly on whether it is evaluated with a Green's function derived from a local or a nonlocal potential. The total energy is decomposed according to Eqs. (16) and (17), which allows us to analyze the different contributions separately using different Green's functions. In the left panel of Fig. 2, we report the Coulomb part of the correlation energy $U_{c}^{G W}[G]$ [given by Eq. (13)] evaluated with Green's functions obtained from PBE, scRPA, HF, and $\operatorname{sc} G W$. The correlation energies evaluated from $\operatorname{sc} G W$ and $G_{0} W_{0} @ \mathrm{HF}$ are in very good agreement, and so are the $U_{c}^{G W}[G]$ derived from the scRPA and PBE Green's functions. However, the correlation energy is significantly larger when the Green's function is derived from a local potential (scRPA and PBE) as compared to a nonlocal one ( $\operatorname{sc} G W$ and $\mathrm{HF}$ ). In fact, the use of a local potential increases the Coulomb energy by almost a factor of 2 in the dissociation region. As analyzed in Ref. [14], this behavior is intimately related to the HOMO-LUMO (or quasiparticle) gap contained in the Green's function. In the $\mathrm{KS}$ system of a molecule with open-shell atoms like the $\mathrm{H}_{2}$ molecule, the gap is largely underestimated and decays exponentially fast to zero with nuclear separation. A nonlocal potential is thus required to reproduce the true quasiparticle gap. Indeed, in the right panel of Fig. 2, we see that the HF and sc $G W$ gaps are closer to the true gap, at least up to $2 \AA$. At larger $R$, the exact full-CI gap quickly reaches a finite value corresponding to the difference between the ionization energy

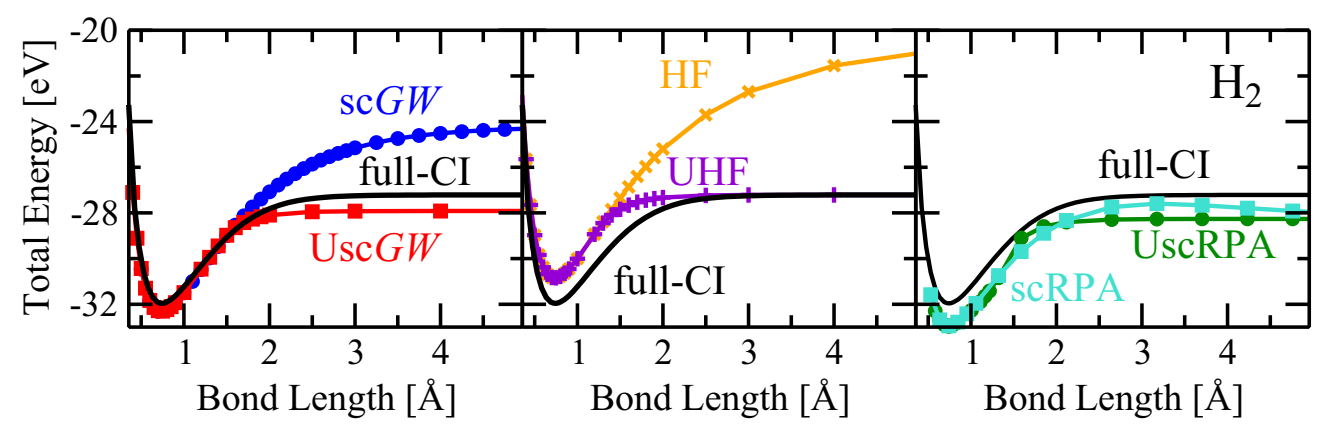

FIG. 1. (Color online) $\mathrm{H}_{2}$ dissociation curves in spin-unrestricted/restricted self-consistent (Usc/sc) $G W$, RPA, and HF. Results are compared to accurate full-CI results from Ref. [41]. 

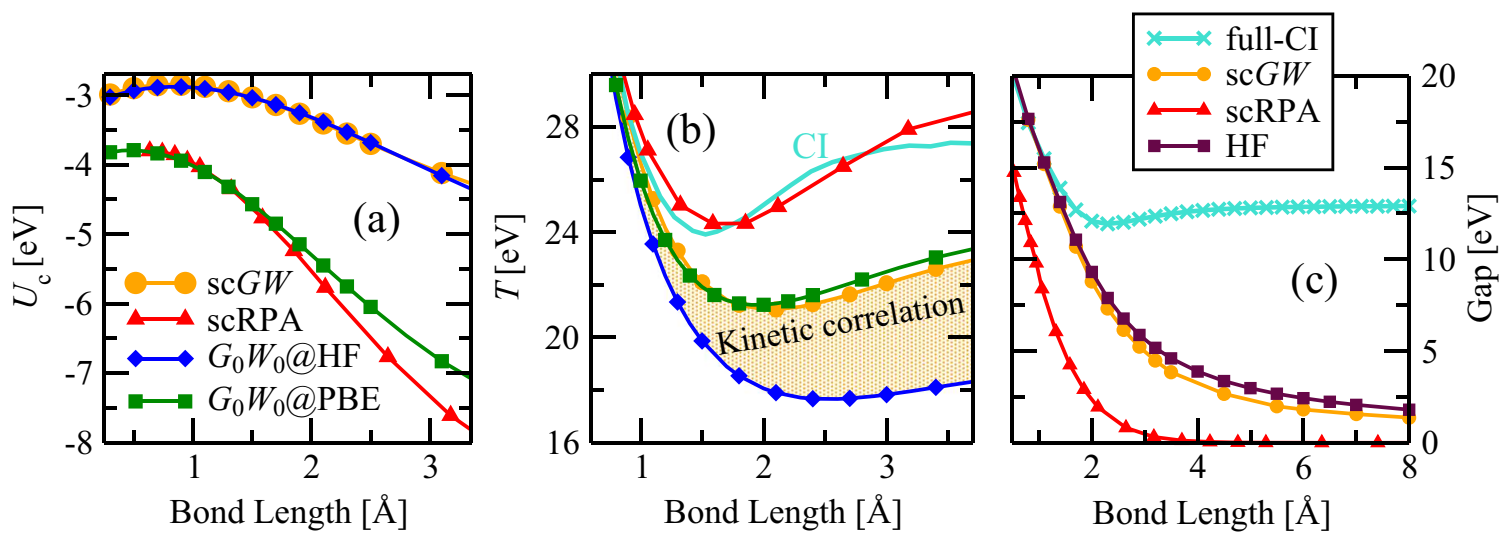

FIG. 2. (Color online) (a) $U_{\mathrm{c}}^{G W}$ evaluated with the KS Green's function in PBE and RPA (denoted $G_{0} W_{0} @ \mathrm{PBE}$ and scRPA) and with $G W$ and HF Green's functions (denoted $G_{0} W_{0} @ \mathrm{HF}$ and $\operatorname{sc} G W$ ). (b) Kinetic energies calculated with different Green's functions as $T\left[G^{G W}\right]$ for $\operatorname{sc} G W, T\left[G^{\mathrm{HF}}\right]$ for $G_{0} W_{0} @ \mathrm{HF}, T\left[G^{\mathrm{PBE}}\right]$ for $G_{0} W_{0} @ \mathrm{PBE}$ and $T\left[G^{\mathrm{RPA}}\right]+T_{\mathrm{c}}\left[G^{\mathrm{RPA}}\right]$ for scRPA. We also report the exact kinetic energy from the full-CI calculations of Ref. [42]. (c) Corresponding HOMO-LUMO gaps.

and the electron affinity of the Hydrogen atom. In contrast, all approximate methods considered here yield a vanishing HOMO-LUMO gap at dissociation. Furthermore, the HF and $\operatorname{sc} G W$ approximations exhibit a very slow convergence (with respect to increasing bond length separation) to the dissociation limit. Since $U_{c}^{G W}$ decays as the square root of the gap [16,32], the large difference in Coulomb correlation energy when using a local and a nonlocal potential is a direct reflection of this slow convergence. The interdependence of the HOMO-LUMO gap and the Coulomb correlation energy $U_{c}^{G W}$ is investigated in more detail in Sec. IV, where the analytical solution of the Green's function for a two-level model of the $\mathrm{H}_{2}$ molecule is presented.

In Fig. 3, the RPA total energy is calculated with a HF Green's function (RPA@HF). The energy is now very close to the self-consistent $G W$ result showing that the nonlocality of the self-energy is a crucial factor for the variational property of the Klein energy expression. The difference between RPA@HF and $G_{0} W_{0} @ \mathrm{HF}$ is exactly $T_{c}\left[G^{\mathrm{HF}}\right]$, which can be seen to be almost constant along the dissociation curve. In the next section, we further analyze kinetic correlation effects.

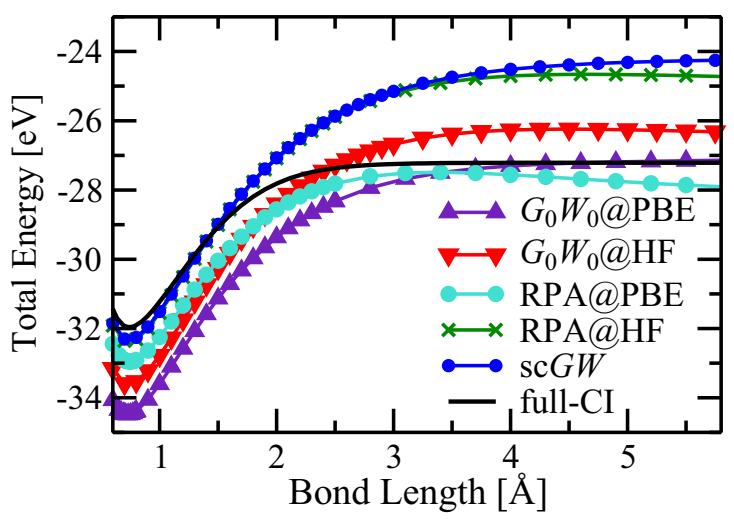

FIG. 3. (Color online) Total energy of $\mathrm{H}_{2}$ evaluated in RPA and $G W$ based on first-order perturbation theory and full-CI results [41].

\section{B. Kinetic energy and density matrix analysis}

In the middle panel of Fig. 2, we report the kinetic contributions to the total energy evaluated in $\mathrm{PBE}, \mathrm{HF}, \operatorname{sc} G W$, and scRPA. The scRPA kinetic energy, defined as the sum of the noninteracting kinetic energy and the kinetic correlation energy [see Eq. (15)], is in close agreement with exact full-CI results [42].

Just like the Coulomb correlation energy $U_{\mathrm{c}}$, also the kinetic energy depends strongly on the input Green's function. However, the $\operatorname{sc} G W\left(T\left[G^{G W}\right]\right)$ and $G_{0} W_{0} @ \mathrm{PBE}\left(T\left[G^{\mathrm{PBE}}\right]\right)$ kinetic energies assume similar values at every $R$. When evaluated with a HF Green function $T\left[G^{\mathrm{HF}}\right]$, on the other hand, the kinetic energy decreases by approximately $15 \%$ $20 \%$. This underestimation can explain almost the most of the difference between $\operatorname{sc} G W$ and $G_{0} W_{0} @ \mathrm{HF}$ around equilibrium (see Fig. 3). However, when stretched, a difference in the external, Hartree and exchange energies also become important indicating that the difference in the density and the density matrix between $\mathrm{HF}$ and $\operatorname{sc} G W$ increases with separation. Indeed, in addition to the importance of screening the Coulomb potential, a qualitative change in the $G W$ ground-state at self-consistency can occur due the frequency dependence of the $G W$ self-energy. Figure 4 shows the first two natural occupation numbers $\left(n_{1}\right.$ and $\left.n_{2}\right)$ of the density

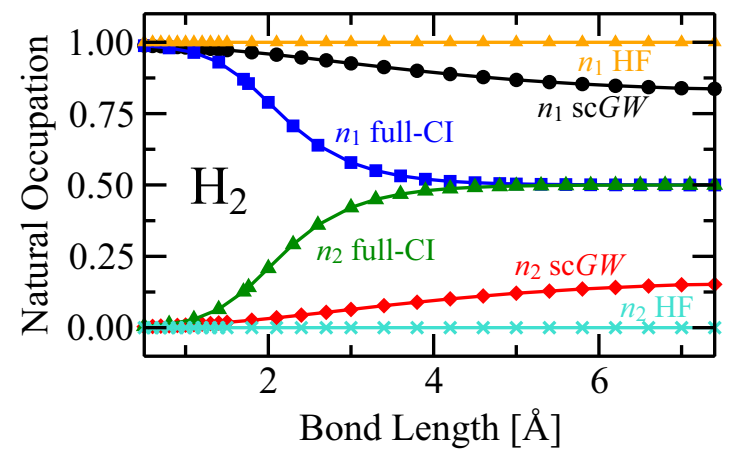

FIG. 4. (Color online) Natural occupation numbers obtained by diagonalizing the density matrix. 
matrix - i.e., the eigenvalues obtained from the density-matrix diagonalization - in the different approximations compared to exact reference data from full-CI calculations. As expected, $\mathrm{HF}$ behaves as an independent particle approximation, i.e., the density matrix occupation numbers are integer at all bond lengths-reflecting the fact that the density matrix is idempotent for noninteracting particle systems. Identical results are obtained for PBE and scRPA. On the other hand, according to exact full-CI calculations, $n_{1}$ and $n_{2}$ should both approach 0.5 in the dissociation limit, due to the multiple Slater-determinant character of the electronic wave function at dissociation. The $G W$ density matrix yields fractional occupation numbers at all bond lengths. Therefore the dynamical $G W$ self-energy leads to interacting ground-state properties that are qualitatively different from the noninteracting particle ground-state of $\mathrm{HF}$, PBE, and scRPA. However, the occupation numbers still largely deviate from the full-CI values, and at $6 \AA n_{1}$ and $n_{2}$ are far from the exact results. In the next section, we illustrate that for a two-level model for $\mathrm{H}_{2}$, the occupation $n_{1}$ and $n_{2}$ reach full degeneracy $\left(n_{1}=n_{2}=0.5\right)$, at dissociation. However, this limit is only reached at very large interatomic separation due to the slow variation of the occupation numbers $\left(\sim \Delta \varepsilon^{1 / 4}\right)$ on the HOMO-LUMO gap $\Delta \varepsilon$. This slow convergence to the dissociation limit, further discussed in Sec. IV, appears a characteristic feature of the $G W$ approximation.

\section{DISSOCIATION LIMIT OF $\mathrm{H}_{2}$ IN A MINIMAL BASIS}

To gain further insight into the dissociation of $\mathrm{H}_{2}$ and, in particular, the dissociation limit $(R \rightarrow \infty)$, in $G W$ and RPA we will study a minimal basis $\mathrm{H}_{2}$ model system described by the HOMO and the LUMO orbitals only. The same model has previously been used to study the dissociation limit of the RPA [31] and the quasiparticle gap in the perturbative $G W$ approximation [52]. More recently, the similar two-site Hubbard model was used to capture qualitative features of $\mathrm{H}_{2}$-dissociation for approximations beyond the RPA [34].

\section{A. Minimal basis model for $\mathbf{H}_{2}$}

At large nuclear separation $R$, the HOMO is approximated by the symmetric linear combination of the atomic orbitals, localized at atom $A$ and $B$,

$$
\varphi_{H}(\mathbf{r})=\frac{1}{\sqrt{2}}\left[\varphi_{A}(\mathbf{r})+\varphi_{B}(\mathbf{r})\right],
$$

and the LUMO is approximated by the corresponding antisymmetric linear combination

$$
\varphi_{L}(\mathbf{r})=\frac{1}{\sqrt{2}}\left[\varphi_{A}(\mathbf{r})-\varphi_{B}(\mathbf{r})\right] .
$$

We can then construct the noninteracting (or KS) time-ordered Green's function

$$
G_{S}\left(\mathbf{r}, \mathbf{r}^{\prime}, \omega\right)=\frac{\varphi_{H}(\mathbf{r}) \varphi_{H}\left(\mathbf{r}^{\prime}\right)}{\omega-\varepsilon_{H}-i \eta}+\frac{\varphi_{L}(\mathbf{r}) \varphi_{L}\left(\mathbf{r}^{\prime}\right)}{\omega-\varepsilon_{L}+i \eta},
$$

where $\varepsilon_{H / L}$ is the HOMO/LUMO eigenvalue. The interacting model Green's function is determined by inverting the Dyson equation [see Eq. (3)]

$$
G\left(\mathbf{r}, \mathbf{r}^{\prime}, \omega\right)=\sum_{i j} \varphi_{i}(\mathbf{r})\left[A^{-1}(\omega)-\Sigma(\omega)\right]_{i j}^{-1} \varphi_{j}\left(\mathbf{r}^{\prime}\right) .
$$

The sum runs over the HOMO and the LUMO and the matrix $A$ is equal to

$$
A^{-1}=\left[\begin{array}{cc}
\omega-\varepsilon_{H}-i \eta & 0 \\
0 & \omega-\varepsilon_{L}+i \eta
\end{array}\right] .
$$

A matrix element of the self-energy is determined from $\Sigma_{i j}(\omega)=\int d \mathbf{r}_{1} d \mathbf{r}_{2} \varphi_{i}\left(\mathbf{r}_{1}\right) \Sigma\left(\mathbf{r}_{1}, \mathbf{r}_{2} ; \omega\right) \varphi_{j}\left(\mathbf{r}_{2}\right)$. In the HF approximation, the self-energy matrix becomes diagonal:

$$
\Sigma=\left[\begin{array}{cc}
\frac{1}{2}\left[U_{0}+U_{1}\right] & 0 \\
0 & \frac{1}{2}\left[U_{0}+3 U_{1}\right]
\end{array}\right]
$$

with

$$
\begin{gathered}
U_{0}=\left\langle\varphi_{A}^{2}|v| \varphi_{A}^{2}\right\rangle=\left\langle\varphi_{B}^{2}|v| \varphi_{B}^{2}\right\rangle \\
U_{1}=\left\langle\varphi_{B}^{2}|v| \varphi_{A}^{2}\right\rangle .
\end{gathered}
$$

Making use of Eq. (24), it is straightforward to evaluate Eq. (22). We find

$$
\begin{aligned}
G_{\mathrm{HF}}\left(\mathbf{r}, \mathbf{r}^{\prime}, \omega\right)= & \frac{\varphi_{H}(\mathbf{r}) \varphi_{H}\left(\mathbf{r}^{\prime}\right)}{\omega-\varepsilon_{H}-\frac{1}{2} U_{0}-\frac{1}{2} U_{1}-i \eta} \\
& +\frac{\varphi_{L}(\mathbf{r}) \varphi_{L}\left(\mathbf{r}^{\prime}\right)}{\omega-\varepsilon_{L}-\frac{1}{2} U_{0}-\frac{3}{2} U_{1}+i \eta}
\end{aligned}
$$

The quasiparticle gap thus increases with $U_{1}$ in the HF approximation. As $R \rightarrow \infty, U_{0}$ remains finite while $U_{1}$ vanishes as $1 / R$. The HF gap therefore reduces to the noninteracting HOMO-LUMO gap in the dissociation limit but decays as $1 / R$, which can be compared to the exponentially fast decay of the noninteracting gap.

\section{B. $G W$ gap in the dissociation limit}

Let us now turn to the $G W$ approximation and evaluate the self-energy with Eq. (21). Hence $\Sigma=i G_{s}[v+v \chi v]$ and

$$
\chi=\chi_{s}+\chi_{s} v \chi
$$

with $\chi_{s}=-i G_{s} G_{s}$. In terms of orbitals and eigenvalues we can write

$$
\chi_{s}=2\left[\frac{f_{s}(\mathbf{r}) f_{s}\left(\mathbf{r}^{\prime}\right)}{\omega-\Delta \varepsilon+i \eta}-\frac{f_{s}(\mathbf{r}) f_{s}\left(\mathbf{r}^{\prime}\right)}{\omega+\Delta \varepsilon-i \eta}\right],
$$

where $f_{s}(\mathbf{r})=\varphi_{H}(\mathbf{r}) \varphi_{L}(\mathbf{r})$ and $\Delta \varepsilon=\varepsilon_{L}-\varepsilon_{H}$. Inserting Eq. (29) into Eq. (28), we find

$$
\chi=2 \frac{\Delta \varepsilon}{\Delta E}\left[\frac{f_{s}(\mathbf{r}) f_{s}\left(\mathbf{r}^{\prime}\right)}{\omega-\Delta E+i \eta}-\frac{f_{s}(\mathbf{r}) f_{s}\left(\mathbf{r}^{\prime}\right)}{\omega+\Delta E-i \eta}\right],
$$

where $\Delta E=\sqrt{\Delta \varepsilon^{2}+4 v_{s} \Delta \varepsilon}$ and $v_{s}=\left\langle f_{s}|v| f_{s}\right\rangle$. 
The correlation part of the self-energy in the $\mathbf{r}$ and $\mathbf{r}^{\prime}$ basis is then easily determined:

$$
\begin{aligned}
\Sigma_{c} & =i \int \frac{d \omega^{\prime}}{2 \pi} G_{s}\left(\omega+\omega^{\prime}\right) v \chi\left(\omega^{\prime}\right) v \\
& =2 \frac{\Delta \varepsilon}{\Delta E}\left[\frac{\lambda_{H H}\left(\mathbf{r}^{\prime}, \mathbf{r}\right)}{\omega+\Delta E-\varepsilon_{H}-i \eta}+\frac{\lambda_{L L}\left(\mathbf{r}^{\prime}, \mathbf{r}\right)}{\omega-\Delta E-\varepsilon_{L}+i \eta}\right]
\end{aligned}
$$

in which

$$
\lambda_{k k}\left(\mathbf{r}^{\prime}, \mathbf{r}\right)=\int d \mathbf{r}_{1} d \mathbf{r}_{2} \varphi_{k}(\mathbf{r}) v\left(\mathbf{r}, \mathbf{r}_{1}\right) f_{s}\left(\mathbf{r}_{1}\right) f_{s}\left(\mathbf{r}_{2}\right) v\left(\mathbf{r}_{2}, \mathbf{r}^{\prime}\right) \varphi_{k}\left(\mathbf{r}^{\prime}\right) .
$$

Alternatively, one may express the self-energy as a matrix in $\varphi_{H}$ and $\varphi_{L}$ :

$$
\Sigma_{c}=\left(\begin{array}{cc}
\frac{\Delta \varepsilon}{2 \Delta E} \frac{\left(U_{0}-U_{1}\right)^{2}}{\omega-\Delta E-\varepsilon_{L}+i \eta} & 0 \\
0 & \frac{\Delta \varepsilon}{2 \Delta E} \frac{\left(U_{0}-U_{1}\right)^{2}}{\omega+\Delta E-\varepsilon_{H}-i \eta}
\end{array}\right),
$$

which is diagonal. In order to solve the Dyson equation with this self-energy, we first notice that we can write

$$
G=G_{\mathrm{HF}}+G_{\mathrm{HF}}\left[\Sigma_{c}+\Delta \Sigma_{\mathrm{HF}}\right] G,
$$

where $\Delta \Sigma_{\mathrm{HF}}$ is the difference between the HF self-energy evaluated with a $G W$ and a HF Green's function. In the following, we will mainly consider the one-shot solution for which this term is zero. In general, this term is not expected to contribute qualitatively. The $G W$ self-energy is frequency dependent, which complicates the extraction of the new poles. In Ref. [52], a perturbative solution was found. However, we can also solve the fully nonlinear equation and after a few manipulations we find in total four solutions:

$$
\begin{aligned}
& \Omega_{ \pm}^{H}=\frac{\varepsilon_{H}^{0}+\varepsilon_{L}+\Delta E}{2} \pm \sqrt{\frac{\left(\Delta E+\varepsilon_{L}-\varepsilon_{H}^{0}\right)^{2}}{4}+k}, \\
& \Omega_{ \pm}^{L}=\frac{\varepsilon_{H}+\varepsilon_{L}^{0}-\Delta E}{2} \pm \sqrt{\frac{\left(\Delta E+\varepsilon_{L}^{0}-\varepsilon_{H}\right)^{2}}{4}+k},
\end{aligned}
$$

where we have defined

$$
k=\frac{\Delta \varepsilon}{2 \Delta E}\left(U_{0}-U_{1}\right)^{2} .
$$

In order to distinguish between the Green's function inserted in the self-energy and the zeroth order Green's function, chosen to be that of the HF in Eq. (34), we have denoted the eigenvalues of the latter $\varepsilon_{H / L}^{0}$ and those of the former $\varepsilon_{H / L}$. Both have the noninteracting form of Eq. (24).

The HOMO and LUMO are easily identified and we find the $G W$ gap:

$$
\begin{aligned}
\Delta= & \Omega_{+}^{L}-\Omega_{-}^{H}=\frac{\varepsilon_{H}+\varepsilon_{L}^{0}-\varepsilon_{H}^{0}-\varepsilon_{L}}{2}-\Delta E \\
& +\sqrt{\frac{\left(\Delta E+\varepsilon_{L}^{0}-\varepsilon_{H}\right)^{2}}{4}+k} \\
& +\sqrt{\frac{\left(\Delta E+\varepsilon_{L}-\varepsilon_{H}^{0}\right)^{2}}{4}+k .}
\end{aligned}
$$

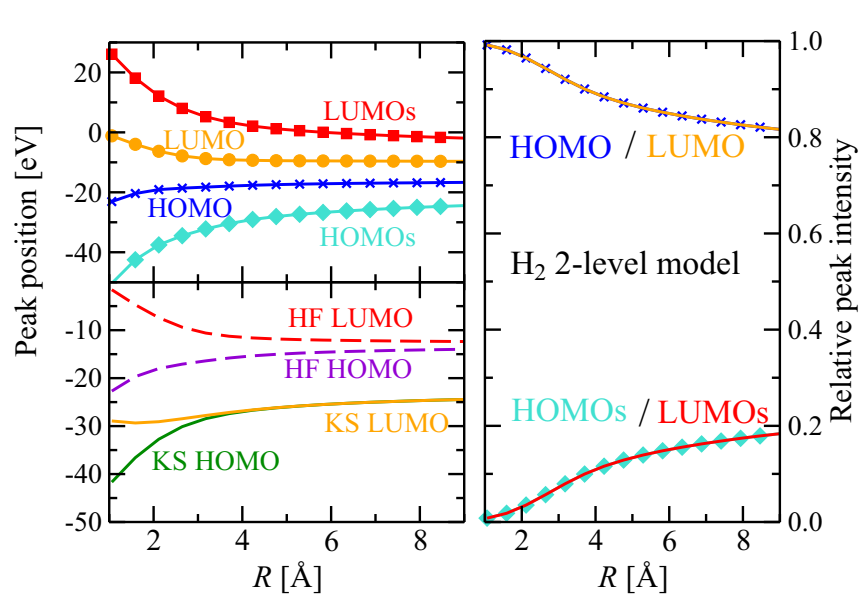

FIG. 5. (Color online) (Left) The four solutions [see Eq. (35)] of quasiparticle excitation energies in the one-shot $G W$ approximation (upper panel) and in the HF and KS-LDA approximations (lower panel) for $\mathrm{H}_{2}$ in a minimal basis. (Right) Quasiparticle amplitudes of the four one-shot $G W$ solutions.

The two additional poles correspond to many-body excitations, i.e., they are accessible only when the ground state is described by more than one Slater determinant. In the two-level model, one of these satellite excitations corresponds to the first excited state of the one-electron system and the other corresponds to the first excited state of the three-electron system.

If the HF Green's function is plugged into the self-energy, we obtain the one-shot $G_{0} W_{0} @ \mathrm{HF}$ solution to the gap:

$$
\Delta=-\Delta E+2 \sqrt{\frac{(\Delta E+\Delta \varepsilon)^{2}}{4}+k}
$$

In order to explicitly see the correction to the HF gap $\Delta \varepsilon$, we can also write Eq. (38) as

$$
\Delta=\Delta \varepsilon+(\Delta E+\Delta \varepsilon)\left[\sqrt{1+2 \frac{\Delta \varepsilon}{\Delta E} \frac{\left(U_{0}-U_{1}\right)^{2}}{(\Delta E+\Delta \varepsilon)^{2}}}-1\right]
$$

from which we can conclude that the correction is always positive. Furthermore, expanding the square root, we recover the perturbative result of Ref. [52]:

$$
\Delta \approx \Delta \varepsilon+\frac{\Delta \varepsilon}{\Delta E} \frac{\left(U_{0}-U_{1}\right)^{2}}{\Delta E+\Delta \varepsilon}
$$

In Fig. 5 (left upper panel), we have plotted the four solutions in the one-shot $G_{0} W_{0} @ \mathrm{HF}$ approximation as a function of $R$. The satellite excitations are denoted with an 's'. According to Eq. (35) all four solutions will converge to the same value in the dissociation limit. The $G_{0} W_{0} @$ HF gap will thus also decay to zero but much slower as compared to HF (left lower panel). This can be attributed to the slowly decaying $1 / \sqrt{R}$ terms that can be extracted from Eq. (38). We again stress that the exact gap should be finite in the dissociation limit (see Fig. 2). 
In order see what is the effect of self consistency we first rewrite Eq. (37) as

$$
\begin{aligned}
\Delta= & \frac{\Delta \varepsilon-\Delta}{2}-\Delta E+\sqrt{\frac{\left(\Delta E+\Delta+\varepsilon_{L}^{0}-\varepsilon_{L}\right)^{2}}{4}+k} \\
& +\sqrt{\frac{\left(\Delta E+\Delta+\varepsilon_{H}-\varepsilon_{H}^{0}\right)^{2}}{4}+k .}
\end{aligned}
$$

As a first step, $\varepsilon_{L}^{0}-\varepsilon_{L}$ and $\varepsilon_{H}-\varepsilon_{H}^{0}$ are set to zero. In this way, it is possible to find a unique solution for $\Delta$ which increases the gap even further. By instead approximating

$$
\varepsilon_{L}^{0}-\varepsilon_{L}=\varepsilon_{H}-\varepsilon_{H}^{0}=\frac{\Delta E+\Delta}{2}-\sqrt{\frac{(\Delta E+\Delta)^{2}}{4}+k},
$$

the gap is similar to the one-shot result. Proceeding to selfconsistency closes the gap further. This result is consistent with the full basis result in Fig. 2.

Let us now determine the quasiparticle amplitudes corresponding to the excitations in Eq. (35). We find

$$
\begin{gathered}
Z_{ \pm}^{H}=\left[\frac{1}{2} \mp \frac{\Delta E+\varepsilon_{L}-\varepsilon_{H}^{0}}{\sqrt{\frac{\left(\Delta E+\varepsilon_{L}-\varepsilon_{H}^{0}\right)^{2}}{4}+k}}\right] \varphi_{H}(\mathbf{r}) \varphi_{H}\left(\mathbf{r}^{\prime}\right), \\
Z_{ \pm}^{L}=\left[\frac{1}{2} \pm \frac{\Delta E+\varepsilon_{L}^{0}-\varepsilon_{H}}{\sqrt{\frac{\left(\Delta E+\varepsilon_{L}^{0}-\varepsilon_{H}\right)^{2}}{4}+k}}\right] \varphi_{L}(\mathbf{r}) \varphi_{L}\left(\mathbf{r}^{\prime}\right) .
\end{gathered}
$$

In the one-shot $G_{0} W_{0} @ \mathrm{HF}$ approximation, these reduce to

$$
\begin{aligned}
Z_{ \pm}^{H} & =\left(\frac{1}{2} \mp P\right) \varphi_{H}(\mathbf{r}) \varphi_{H}\left(\mathbf{r}^{\prime}\right), \\
Z_{ \pm}^{L} & =\left(\frac{1}{2} \pm P\right) \varphi_{L}(\mathbf{r}) \varphi_{L}\left(\mathbf{r}^{\prime}\right),
\end{aligned}
$$

where

$$
P=\frac{1}{4} \frac{\Delta E+\Delta \varepsilon}{\sqrt{\frac{(\Delta E+\Delta \varepsilon)^{2}}{4}+k}} .
$$

The weight of the HOMO and LUMO excitations thus decreases with nuclear separation whereas the weights of the many-body excitations increases (see right panel of Fig. 5). It is easy to see that $P$ tends to zero as $\Delta \varepsilon^{1 / 4}$ with nuclear separation and thus all four excitations become degenerate and acquire the same weight of $1 / 2$ in the dissociation limit. This means that the density matrix is correctly described in terms of natural occupation numbers in the dissociation limit. However, due to the slow $\Delta \varepsilon^{1 / 4} \propto R^{-1 / 4}$ decay, the dissociation limit is reached only at very large $R$. The same behavior is found in the full basis results in Fig. 4.

The time-ordered one-shot $G W$ Green's function can finally be summarized as

$$
\begin{aligned}
G_{G W}= & Z_{-}^{H} \frac{\varphi_{H}(\mathbf{r}) \varphi_{H}\left(\mathbf{r}^{\prime}\right)}{\omega-\Omega_{-}^{H}-i \eta}+Z_{+}^{H} \frac{\varphi_{H}(\mathbf{r}) \varphi_{H}\left(\mathbf{r}^{\prime}\right)}{\omega-\Omega_{+}^{H}+i \eta} \\
& +Z_{-}^{L} \frac{\varphi_{L}(\mathbf{r}) \varphi_{L}\left(\mathbf{r}^{\prime}\right)}{\omega-\Omega_{-}^{L}-i \eta}+Z_{+}^{L} \frac{\varphi_{L}(\mathbf{r}) \varphi_{L}\left(\mathbf{r}^{\prime}\right)}{\omega-\Omega_{+}^{L}+i \eta} .
\end{aligned}
$$

\section{C. $G W$ and RPA correlation energy at dissociation}

We will now use the HF/KS and the one-shot $G W$ Green's functions to study the correlation energy in the dissociation limit. To this purpose, we need to construct the zeroth-order polarization propagator. For a HF/KS Green's function this was done in Eq. (29). With the many-body $G W$ Green's function of Eq. (48), we find in total four poles with energies and oscillator strengths:

$$
\begin{aligned}
& 1: \Delta \varepsilon_{1}=\Omega_{+}^{H}-\Omega_{-}^{H}, f_{1}(\mathbf{r})=\sqrt{\frac{1}{4}-P^{2}} \varphi_{H}(\mathbf{r}) \varphi_{H}(\mathbf{r}), \\
& 2: \Delta \varepsilon_{2}=\Omega_{+}^{L}-\Omega_{-}^{L}, f_{2}(\mathbf{r})=\sqrt{\frac{1}{4}-P^{2}} \varphi_{L}(\mathbf{r}) \varphi_{L}(\mathbf{r}), \\
& 3: \Delta \varepsilon_{3}=\Omega_{+}^{H}-\Omega_{-}^{L}, f_{3}(\mathbf{r})=\left(\frac{1}{2}-P\right) \varphi_{L}(\mathbf{r}) \varphi_{H}(\mathbf{r}), \\
& 4: \Delta \varepsilon_{4}=\Omega_{+}^{L}-\Omega_{-}^{H}, f_{4}(\mathbf{r})=\left(\frac{1}{2}+P\right) \varphi_{L}(\mathbf{r}) \varphi_{H}(\mathbf{r}) .
\end{aligned}
$$

Both the correlation energy in RPA and the correlation energy in $\operatorname{sc} G W$ contain the term $-\int \operatorname{Tr}\left\{v \chi_{0}\right\}$. It is easy to see that this term gives identical results in the dissociation limit independently of which of the above $G$ is used for constructing $\chi_{0}$. In both cases, we find

$$
-\lim _{R \rightarrow \infty} \int_{0}^{\infty} \frac{d \omega}{2 \pi} \operatorname{Tr}\left[v \chi_{0}(i \omega)\right]=-\frac{U_{0}}{2} .
$$

This is exactly equal to the static correlation error of the $\mathrm{EXX} / \mathrm{HF}$ functional $\left(U_{0} / 2\right)$ and hence this term cancels this error in both RPA and $G W$. We will now show that the second term of the correlation energy $-\int \operatorname{Tr}\left(v \chi_{H}\right)$ gives zero contribution in the dissociation limit. The interacting polarization propagator within $\mathrm{HF} / \mathrm{KS}$ is given by Eq. (30) and it therefore decays as $\sqrt{\Delta \varepsilon}$ as $R \rightarrow \infty$. Performing the $\lambda$ integral does not change this result. This explains the difference in rate at which the asymptotic limit is reached by inserting HF or a KS Green's function.

Now let us see what we find when we use the one-shot $G W$ Green's function. Inserting this response function into the RPA equations leads to a $4 \times 4$ matrix. We notice, however, that this matrix can be written in terms of two sub-blocks, one in terms of the first and second excitations and the second in terms of the third and fourth excitations. We thus have to diagonalize two $2 \times 2$ matrices independently. For example, in terms of excitations 3 and 4 , we find

$$
\left(\begin{array}{cc}
\Delta \varepsilon_{3}^{2}+v_{33} & v_{34} \\
v_{43} & \Delta \varepsilon_{4}^{2}+v_{44}
\end{array}\right),
$$

where

$$
v_{i j}=\left\langle\tilde{f}_{i}|v| \tilde{f}_{j}\right\rangle, \quad \tilde{f}_{j}=2 \sqrt{\Delta \varepsilon_{j}} f_{j} .
$$


The eigenvalues $\lambda_{ \pm}$and eigenvectors $\left(d_{1}^{ \pm} d_{2}^{ \pm}\right)$of this matrix are

$$
\begin{aligned}
\lambda_{ \pm}= & \frac{\Delta \varepsilon_{3}^{2}+v_{33}+\Delta \varepsilon_{4}^{2}+v_{44}}{2} \\
& \pm \sqrt{\frac{\left(\Delta \varepsilon_{3}^{2}+v_{33}-\Delta \varepsilon_{4}^{2}-v_{44}\right)^{2}}{4}+v_{34}^{2},} \\
d_{1}^{+}= & \frac{v_{34}}{\sqrt{v_{34}^{2}+\left(\lambda_{+}-\Delta \varepsilon_{3}^{2}-v_{33}\right)^{2}}}, \\
d_{2}^{+}= & \frac{\lambda_{+}-\Delta \varepsilon_{3}^{2}-v_{33}}{\sqrt{v_{34}^{2}+\left(\lambda_{+}-\Delta \varepsilon_{3}^{2}-v_{33}\right)^{2}}}, \\
d_{1}^{-}= & \frac{v_{34}}{\sqrt{v_{34}^{2}+\left(\lambda_{-}-\Delta \varepsilon_{3}^{2}-v_{33}\right)^{2}}}, \\
d_{2}^{-}= & \frac{\lambda-\Delta \varepsilon_{3}^{2}-v_{33}}{\sqrt{v_{34}^{2}+\left(\lambda_{-}-\Delta \varepsilon_{3}^{2}-v_{33}\right)^{2}}},
\end{aligned}
$$

which yields the new oscillator strengths

$$
\begin{aligned}
& \tilde{f}_{3}=2 d_{1}^{+} \sqrt{\frac{\Delta \varepsilon_{3}}{\sqrt{\lambda_{+}}}} f_{3}+2 d_{2}^{+} \sqrt{\frac{\Delta \varepsilon_{4}}{\sqrt{\lambda_{+}}}} f_{4}, \\
& \tilde{f}_{4}=2 d_{1}^{-} \sqrt{\frac{\Delta \varepsilon_{3}}{\sqrt{\lambda_{-}}}} f_{3}+2 d_{2}^{-} \sqrt{\frac{\Delta \varepsilon_{4}}{\sqrt{\lambda_{-}}}} f_{4} .
\end{aligned}
$$

It is easy to see that also these tend to zero in the dissociation limit. Thus, even when inserting a one-shot $G W$ Green's function the term $-\int \operatorname{Tr}\left(v \chi_{H}\right)$ will tend to zero in the dissociation limit, suggesting that the energy will be similar to that found in RPA or RPA@ $@ \mathrm{HF}$. From the full $\mathrm{H}_{2} G W$ dissociation curves, we can thus not exclude the possibility that the $G W$ curve exhibits a very extended local maximum (i.e., a bump) that decays to the correct dissociation limit a very large interatomic separation. We will discuss this topic more in the next section.

\section{FRACTIONAL SPIN AND FRACTIONAL CHARGE ERRORS}

The fractional spin and fractional charge errors have been rigorously defined in Ref. [53]. The study of these errors consists in treating the atoms in the dissociation limit independently but by means of ensembles that allow for fractional charge and spin. If the density that minimizes the energy is integrated to a fractional number of particles on the individual atoms, the functional suffers a fractional charge or delocalization error and if an atom with fractional spin has a different energy from an atom with integer spin the functional suffers a fractional spin or static correlation error.

Within DFT and RPA a generalization in terms of ensembles was made in Ref. [35]. However, in $G W$, and MBPT, such a procedure is less straightforward [54]. In this work, we have therefore used a more indirect approach to estimate fractional charge and fractional spin errors in $G W$ based on the calculation of spin-unrestricted dissociation curves and dipole moments of heteroatomic dimers.

\section{A. Spin-unrestricted dissociation}

We have seen that there is a fundamental difference between RPA and $G W$ in how they deal with static correlation at large or intermediate distances. RPA is almost free from static correlation errors and describes correctly the dissociation of covalent bonds. On the other hand, $\operatorname{sc} G W$ overestimates the total energy at dissociation, with an error comparable to the PBE functional. It is well known that by breaking the spin symmetry, it is possible to simulate charge localization and avoid static correlation errors in near degeneracy situations. However, despite the improved description of the total energy, the spin-unrestricted ground-state is unphysical since it has the wrong spin-density.

The spin symmetry breaking is achieved by localizing the spin-up and spin-down electrons on different atoms in the initialization of the calculation. For bond lengths close to the equilibrium one, this procedure yields identical wave functions and total energies as for spin-restricted calculations. However, for larger bond lengths, the total energy obtained from unrestricted calculations lies at a lower energy than the restricted one.

In Fig. 1, we report spin-unrestricted self-consistent (Usc) calculations of the $\mathrm{H}_{2}$-dissociation curve in RPA, $G W$, and HF. Figure 1 illustrates that UscRPA is similar to spin-restricted scRPA across the entire dissociation regionconsistent with the fact that RPA has no fractional spin error [35]. The lack of fractional spin error ensures that the spinrestricted and unrestricted curves converge to the same value asymptotically.

On the contrary, the $\operatorname{Usc} G W$ dissociation curve differs considerably from the spin-restricted $\operatorname{sc} G W$ curve at large internuclear distances and improves the dissociation region as expected. This picture confirms that at finite but large $R$ (i.e., at bond lengths significantly larger than the point of atomization in the exact treatment), sc $G W$ suffers large fractional spin error. However, when inserting a HF Green's function in the $G W$ (Klein) energy expression a spurious maximum around $4 \AA$ can be observed (see Fig. 3), indicating that the energy eventually may improve-albeit at very large separation. These results are consistent with the two-level model studied in Sec. IV, where we show that the $G W$ energy evaluated with $G_{\mathrm{HF}}$ converges to the RPA energy (i.e., to the correct dissociation energy) in the limit of large separation but much slower than in RPA due to the slow decay of the $\mathrm{HF}$ quasiparticle gap. It is further shown that inserting a one-shot $G W$ Green's function does not change this result, which suggests that the $\operatorname{sc} G W$ total energy might present an extended local maximum (or bump), which decreases to the exact dissociation limit for $R \rightarrow \infty$. This bump is a well known feature of the RPA functional [31] and also in RPA it does not disappear with self-consistency [14]. From Fig. 1, we can further conclude that spin-unrestricted $G W$ and RPA calculations do not exhibit spurious maxima.

\section{B. Dipole moment of $\mathrm{LiH}$}

The problem of delocalization or fractional charge error is related to the tendency of approximate functionals to 


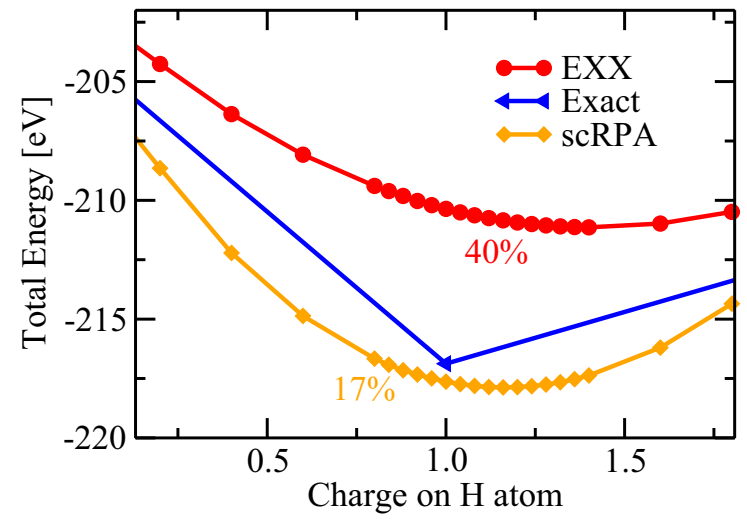

FIG. 6. (Color online) Fractional charge analysis of the $\mathrm{LiH}$ molecule. The total energy as a function of fractional charge on the $\mathrm{H}$ atom. These curves are obtained by adding the fractional charge curves of the independent atoms in such a way that the total number of electrons is four.

spread out, i.e., delocalize, the electrons in the system due to an insufficient treatment of the Coulomb repulsion. This problem gives rise to large errors when looking at response properties [55,56], but also results in an error in the total energy due to an incorrect electron distribution in the ground state.

A very simple system, which clearly reveals the delocalization error, is the stretched $\mathrm{LiH}$ molecule. It is a heteronuclear system and therefore very sensitive to the ability of the functional to localize the electrons in the dissociation process. Molecules should dissociate into neutral atoms. However, many approximations produce fractionally charge atoms in the dissociation limit of heteronuclear systems composed of open shell atoms [35] and the amount of fractional charge can be seen as a measure of the delocalization error. Obviously, homonuclear systems do not exhibit this behavior in the dissociation limit due to symmetry. This does, however, not mean that there is no delocalization problem. Also at finite separation the electrons can be too delocalized resulting in, e.g., a slowly converging potential energy curve.

A way to determine this error without actually calculating the energy of the infinitely stretched molecule is to do a fractional charge analysis. In DFT, the functional is extended to ensemble densities that allows for noninteger number of electrons. The energy is then calculated as a function of particle number. Whereas the exact functional exhibits cusps at the integers and is linear between the integers most approximations produces smooth curves with a large curvature. To calculate the energy of the molecule at infinite separation, the atomic curves are added and the minimum is located. The exact functional minimizes nonanalytically at the integer which ensures neutral dissociation. However, the usually smooth approximate curves lead to a shift in the minimum as we will see below.

The generalization of the RPA to ensemble densities was done in Ref. [35]. In Fig. 6, we have plotted the energy as a function of the total (fractional) number of electrons on the $\mathrm{H}$ atom. We see that EXX minimizes smoothly at around 1.4 electrons due to the nonlinear behavior of the EXX functional. RPA is seen to reduce this error to $17 \%$ but we still see a rather large curvature in the RPA.

In MBPT it is much more difficult to disentangle the dissociated atoms and treat them as fractionally charged isolated systems due to the frequency-dependent self-energy. To circumvent this complication we have studied the problem by looking directly at the density of the stretched molecule via the dipole moment. In Fig. 7, we compare $G W$, HF, and PBE. For comparison, we also report coupled-cluster singles doubles (CCSD) dipole moments [57] at the complete basis-set limit performed with ORCA code package [46]. At around $3.5 \AA$, the CCSD dipole moment exhibits a rather sharp transition from a finite value to zero. This indicates the breaking of the molecular bond into two neutral atoms. None of the approximations studied here is able to capture this transition. Instead, the dipole moment steadily increases with separation due to the delocalization error. By dividing the dipole moment with the distance, we estimate the fractional charge error at large (but finite) separation. At $6 \AA$, we find that the residual charge left on the $\mathrm{Li} / \mathrm{H}$ fragments at dissociation amounts to $20 \%$ of an electron in $\operatorname{sc} G W, 35 \%$ in HF, and $18 \%$ in PBE. The problem of delocalization is thus not reduced in the many body framework and is rather substantial in the $G W$ approximation-similar to the RPA. The density matrix analysis for $\mathrm{H}_{2}$ (Sec. IV) in a minimal basis shows that we correctly obtain half-occupation of the natural orbitals in the dissociation limit. This suggest that also in $\mathrm{LiH}$ we may dissociate correctly in $G W$. However, again this would occur at an unphysically large $R$.

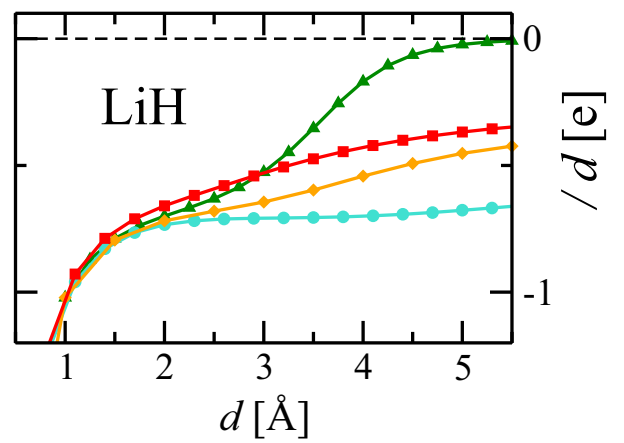

FIG. 7. (Color online) (Left) The dipole moment of the LiH molecule as a function of bond distance in HF, PBE, sc $G W$, and accurate CCSD calculations. (Right) The dipole moment divided by distance in the same approximations gives an estimate of their fractional charge error. 


\section{SUMMARY AND CONCLUSIONS}

In summary, we have presented a general assessment of the $\operatorname{sc} G W$ and scRPA approximations for ground-state properties of prototype diatomic molecules composed of open shell atoms. The analysis of the different total energy contributions reveals large differences between the use of a nonlocal and frequency-dependent self-energy in the $G W$ approximation and the use of a static and local potential in RPA, both evaluated at self-consistency.

Nonlocality leads to smaller Coulomb correlation energies due to the larger gap between occupied and empty states. From the analysis of the density matrix, we deduce that the $\operatorname{sc} G W$ ground state is not representable in terms of a single Slater determinant. The noninteger eigenvalues of the density matrix illustrates a transition from a noninteracting to an interacting ground state at self-consistency. Furthermore, screening effects within $G W$ increase the kinetic energy as compared to Hartree-Fock. A similar effect can be achieved using the static local RPA potential.

The solution of a two-level model of the $\mathrm{H}_{2}$ molecule at dissociation complements our first-principles calculations and allows to investigate the limit of infinite interatomic separation. For this simplified model, both $G W @ \mathrm{HF}$ and RPA reach the correct dissociation limit. However, within $G W$ the bond-breaking regime is reached at an unphysical large bond length.

For the case of LiH, we have shown that both scRPA and $\operatorname{sc} G W$ are affected by strong delocalization or fractional charge errors. In RPA this leads to the dissociation of $\mathrm{LiH}$ in non-neutral fragments.

The sc $G W$ dissociation curve can be considerably improved by resorting to spin-unrestricted calculations, whereas in scRPA, spin-unrestricted, and restricted coincide at dissociation. This demonstrates that scRPA — similarly to perturbative RPA - is free from fractional-spin error. On the other hand, sc $G W$ suffers from a large static correlation or fractional spin errors that is responsible for the qualitatively incorrect description of the dissociation region.

\section{ACKNOWLEDGMENTS}

AR acknowledges financial support by the European Research Council Advanced Grant DYNamo (ERC-2010AdG-267374), European Commission project CRONOS (Grant number 280879-2 CRONOS CP-FP7), Spanish Grant (FIS2013-46159-C3-1-P) and Grupo Consolidado UPV/EHU del Gobierno Vasco (IT578-13). PR acknowledges financial support from the Academy of Finland through its Centres of Excellence Program (Project No. 251748).
[1] A. L. Fetter and J. D. Walecka, Quantum Theory of ManyParticle Systems (Dover, New York, 2003).

[2] P. Hohenberg and W. Kohn, Phys. Rev. 136, B864 (1964).

[3] W. Kohn and L. J. Sham, Phys. Rev. 140, A1133 (1965).

[4] R. M. Dreizler and E. K. U. Gross, Density Functional Theory (Springer, Berlin, 1990).

[5] L. Hedin, Phys. Rev. 139, A796 (1965).

[6] D. C. Langreth and J. P. Perdew, Phys. Rev. B 15, 2884 (1977).

[7] W. G. Aulbur, L. Jönsson, and J. W. Wilkins, Solid State Phys. 54, 1 (2000).

[8] G. Onida, L. Reining, and A. Rubio, Rev. Mod. Phys. 74, 601 (2002).

[9] P. Rinke, A. Qteish, J. Neugebauer, C. Freysoldt, and M. Scheffler, New J. Phys. 7, 126 (2005).

[10] A. Hesselmann and A. Görling, Mol. Phys. 108, 359 (2010).

[11] H. Eshuis, J. E. Bates, and F. Furche, Theor. Chem. Acc. 131, 1084 (2012).

[12] X. Ren, P. Rinke, C. Joas, and M. Scheffler, J. Mater. Sci. 47, 7447 (2012).

[13] M. Hellgren and U. von Barth, Phys. Rev. B 76, 075107 (2007).

[14] F. Caruso, D. R. Rohr, M. Hellgren, X. Ren, P. Rinke, A. Rubio, and M. Scheffler, Phys. Rev. Lett. 110, 146403 (2013).

[15] A. Klein, Phys. Rev. 121, 950 (1961).

[16] N. E. Dahlen, R. van Leeuwen, and U. von Barth, Phys. Rev. A 73, 012511 (2006).

[17] M. S. Hybertsen and S. G. Louie, Phys. Rev. Lett. 55, 1418 (1985).

[18] T. Olsen and K. S. Thygesen, Phys. Rev. B 87, 075111 (2013).

[19] J. Harl, L. Schimka, and G. Kresse, Phys. Rev. B 81, 115126 (2010).
[20] F. Furche, J. Chem. Phys. 129, 114105 (2008).

[21] F. Aryasetiawan, T. Miyake, and K. Terakura, Phys. Rev. Lett. 88, 166401 (2002).

[22] A. Hesselmann and A. Görling, Mol. Phys. 109, 2473 (2011).

[23] A. Stan, N. E. Dahlen, and R. van Leeuwen, J. Chem. Phys. 130, 114105 (2009).

[24] F. Caruso, Ph.D. Thesis, Freie Universität Berlin, 2013.

[25] F. Caruso, P. Rinke, X. Ren, M. Scheffler, and A. Rubio, Phys. Rev. B 86, 081102 (2012).

[26] A. Stan, N. E. Dahlen, and R. van Leeuwen, Europhys. Lett. 76, 298 (2006).

[27] K. S. Thygesen and A. Rubio, Phys. Rev. B 77, 115333 (2008).

[28] J. Klimes and G. Kresse, J. Chem. Phys. 140, 054516 (2014).

[29] P. Bleiziffer, A. Hesselmann, and A. Görling, J. Chem. Phys. 139, 084113 (2013).

[30] F. Caruso, V. Atalla, X. Ren, A. Rubio, M. Scheffler, and P. Rinke, Phys. Rev. B 90, 085141 (2014).

[31] M. Fuchs, Y.-M. Niquet, X. Gonze, and K. Burke, J. Chem. Phys. 122, 094116 (2005).

[32] A. Hesselmann and A. Görling, Phys. Rev. Lett. 106, 093001 (2011).

[33] P. Mori-Sánchez, A. J. Cohen, and W. Yang, Phys. Rev. A 85, 042507 (2012).

[34] T. Olsen and K. S. Thygesen, J. Chem. Phys. 140, 164116 (2014).

[35] M. Hellgren, D. R. Rohr, and E. K. U. Gross, J. Chem. Phys. 136, 034106 (2012).

[36] V. Galitskii and A. Migdal, Sov. Phys. JETP 7, 96 (1958).

[37] J. M. Luttinger and J. C. Ward, Phys. Rev. 118, 1417 (1960).

[38] C.-O. Almbladh, U. V. Barth, and R. V. Leeuwen, Int. J. Mod. Phys. B 13, 535 (1999). 
[39] U. von Barth, N. E. Dahlen, R. van Leeuwen, and G. Stefanucci, Phys. Rev. B 72, 235109 (2005).

[40] T. Grabo, T. Kreibich, S. Kurth, and E. K. U. Gross, in Strong Coulomb Correlations in Electronic Structure Calculations: Beyond the Local Density Approximation, edited by V. Anisimov (Gordon and Breach, Amsterdam, 2000).

[41] L. Wolniewicz, J. Chem. Phys. 99, 1851 (1993).

[42] R. van Leeuwen, O. Gritsenko, and E. Baerends, Density Functions, edited by R. F. Nalewajski, Topics in Current Chemistry Vol. 180 (Springer, Berlin, 1996), p. 107.

[43] V. Blum, R. Gehrke, F. Hanke, P. Havu, V. Havu, X. Ren, K. Reuter, and M. Scheffler, Comput. Phys. Commun. 180, 2175 (2009).

[44] X. Ren, P. Rinke, V. Blum, J. Wieferink, A. Tkatchenko, A. Sanfilippo, K. Reuter, and M. Scheffler, New J. Phys. 14, 053020 (2012).

[45] F. Caruso, P. Rinke, X. Ren, A. Rubio, and M. Scheffler, Phys. Rev. B 88, 075105 (2013).

[46] F. Neese, Wiley Interdiscip. Rev.: Comput. Mol. Sci. 2, 73 (2012).
[47] J. Thom H. Dunning, J. Chem. Phys. 90, 1007 (1989).

[48] L. J. Sham and M. Schlüter, Phys. Rev. Lett. 51, 1888 (1983).

[49] M. E. Casida, Phys. Rev. A 51, 2005 (1995).

[50] Y. M. Niquet, M. Fuchs, and X. Gonze, Phys. Rev. A 68, 032507 (2003).

[51] J. P. Perdew, K. Burke, and M. Ernzerhof, Phys. Rev. Lett. 77, 3865 (1996).

[52] F. Aryasetiawan, R. Sakuma, and K. Karlsson, Phys. Rev. B 85, 035106 (2012).

[53] A. J. Cohen, P. Mori-Sánchez, and W. Yang, Science 321, 792 (2008).

[54] F. Bruneval, Phys. Rev. Lett. 103, 176403 (2009).

[55] S. J. A. van Gisbergen, P. R. T. Schipper, O. V. Gritsenko, E. J. Baerends, J. G. Snijders, B. Champagne, and B. Kirtman, Phys. Rev. Lett. 83, 694 (1999).

[56] S. Kümmel, L. Kronik, and J. P. Perdew, Phys. Rev. Lett. 93, 213002 (2004).

[57] A. Szabo and N. S. Ostlund, Modern Quantum Chemistry: Introduction to Advanced Electronic Structure Theory (McGraw-Hill, New York, 1989). 